\title{
Protein-mediated Morphological Regulation of Magnetite Crystal in Magnetotactic Bacteria
}

\author{
A. ARAKAKI, A. YAMAGISHI, and T. MATSUNAGA
}

Division of Biotechnology, and Life Science, Institute of Engineering, Tokyo University of Agriculture and Technology, 2-24-16

Naka-cho, Koganei, Tokyo 184-8588, Japan.

\begin{abstract}
Magnetotactic bacteria are microorganisms that produce magnetic particles surrounded by a lipid bilayer membrane with proteins. Since the size and morphology of these particles are uniform, it is thought that magnetic particles are morphologically controlled within the bacteria. The Mms6 protein was isolated from the surface of cubo-octahedral magnetic particles formed in the Magnetospirillum magneticum strain AMB-1. By using Mms6, cubo-octahedral magnetic particles were synthesized in vitro. Furthermore, the analysis of the mms6 gene-deletion mutant of magnetotactic bacteria indicated that Mms6 plays a role in the in vivo regulation of magnetic crystal morphology during crystal growth. Thus, Mms6 can be used to synthesize morphologically controlled magnetic materials under normal temperature and pressure conditions.
\end{abstract}

Key Words: Magnetic particle, Biomineralization, Magnetotactic bacteria, Mms6 protein, Morphological control

\section{INTRODUCTION}

Biominerals are synthesized under normal temperature and pressure conditions by living organisms. Thus, the biogenesis process, called biomineralization, is an attractive strategy, which does not require a large amount of energy or harsh conditions for the synthesis of materials. Magnetotactic bacteria synthesize magnetite nanoparticles of $\mathrm{Fe}_{3} \mathrm{O}_{4}$ within cells [1]. These particles are assembled into a chain, thus enabling the bacterial cells to swim along magnetic field lines. Various species of magnetotactic bacteria produce magnetic particles of differing shapes (e.g., cubo-octahedral [2-4], elongated hexagonal prism [5], and tooth- or bullet-shaped [6-7]). Considering these findings, it is possible that magnetotactic bacteria possess the regulation mechanisms of magnetite crystal morphologies, for which specifically produced proteins in individual species are likely used. We have previously isolated the Mms6 protein that is specifically localized on the magnetic particles in magnetotactic bacteria [8]. This protein was found to play an important role in the morphological control of magnetic particles in this organism. In this paper, we introduce an application for, and basic studies of, Mms6, which regulates the morphology of magnetic materials.

\section{IDENTIFICATION OF CRYSTAL REGULATOR PROTEIN, MMS6}

Magnetospirillum magneticum strain AMB-1 synthesizes the cubo-octahedral magnetite crystal, which is approximately $50 \mathrm{~nm}$ in diameter in cells (Fig. 1). The coercivity of magnetite from strain AMB-1 was determined to be approximately $8 \mathrm{mT}(6.4 \mathrm{kA} / \mathrm{m})$ at room temperature, and that of chemically synthesized magnetite (diameter 18-45 nm) was approximately $6 \mathrm{mT}$ ( 4.8 $\mathrm{kA} / \mathrm{m}$ ) [9]. The first genome-wide sequencing of a magnetotactic bacterium was completed using this strain [10]. The genome information obtained enabled the proteomic analysis of the magnetosome protein and the transcriptomic analysis of the AMB-1 strain [11-12]. Therefore, the strain is now used as a model organism to study magnetite biomineralization in magnetotactic bacteria. The Mms6 protein was discovered from the surfaces of magnetic particles in AMB-1 [8]. The lipid bilayer and major proteins surrounding bacterial magnetic particles were first removed using a urea solution. Secondly, the particles were boiled with a $1 \%$ SDS solution in order to extract the proteins that remained on the particles. Using 2-dimensional gel electrophoresis, 4 spots were detected from the protein fraction extracted by the $1 \%$ SDS solution. From N-terminal amino acid sequence analysis of these spots at $5 \mathrm{kDa}(\mathrm{pI} 6.1), 6 \mathrm{kDa}(\mathrm{pI} 4.5), 7 \mathrm{kDa}(\mathrm{pI} 5.9)$, and $13 \mathrm{kDa}(\mathrm{pI}$ 
7.2), the proteins showed high homology [8]. They were named Mms5, Mms6, Mms7, and Mms13, respectively, and were localized on the surfaces of magnetic particles. In particular, amino acid sequences of the Mms6 N-terminal region are contained by a GL hydrophobic sequence (repeat of glycine-leucine), and the C-terminal region is a hydrophilic region with acidic amino acids (i.e., asparatic acid and glutamic acid) (Fig. 2). In addition, competitive iron-binding analysis showed that the Mms6 protein had iron-binding activity [8]. When the magnetic particles were synthesized by coprecipitation in the presence of Mms6, the spherical magnetic particles, with sizes ranging from 20-30 nm, were observed by transmission electron microscopy (TEM) [8]. On the other hand, the nonhomogeneous magnetic particles were synthesized in the absence of Mms6. These results suggest that Mms6 can act as a regulator of magnetite crystal morphology in AMB-1, and other Mms proteins have a complementary function.

MGEMEREGAAAKAGAAKTGAAKTGTV AKTGIAAKTGVATAVAAPAAPANVAAAQ GAGTKVALGAGKAAAGAKVVGGTIWTG KGLGLGLGLGLGAWGPIILGVVGAGAV YAYMKSRDIESAQSDEEVELRDALA

Fig. 2 Amino acid sequence of the Mms6 protein. gray, putative signal sequence; blue, GL repeated region; red, acidic amino acid residues; under line, hypothetical mature Mms6 protein.

\section{CHEMICAL MAGNETITE SYNTHESIS USING MMS6 PROTEIN}

The Mms6 protein was likely involved in the synthesis of magnetic particles in strain AMB-1. By using this protein, we succeeded in controlling the morphologies of magnetite crystals in vitro under ordinary temperature and pressure conditions [13]. When the magnetic particles were synthesized with Mms6 by partial oxidation, which is known to produce well-defined octahedral magnetite crystals, the narrow size and shape of the magnetic particles were observed by TEM [13]. The morphology of these particles was similar to the magnetic particles produced in the bacteria. The high-resolution TEM analysis revealed that these crystals consisted of $\left\{\begin{array}{lll}1 & 0 & 0\end{array}\right\}$ and $\left\{\begin{array}{lll}1 & 1 & 1\end{array}\right\}$ crystal faces (Fig. 3) [13]. On the other hand, the magnetic crystals synthesized without Mms6 were octahedral in shape and consisted of only the $\left\{\begin{array}{lll}1 & 1 & 1\end{array}\right\}$ crystal face (Fig. 3) [13]. This result clearly indicates that Mms6 regulates the morphology of

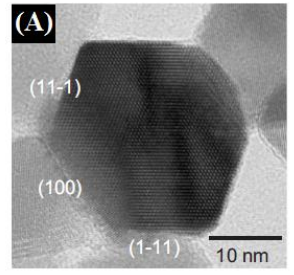

(B)

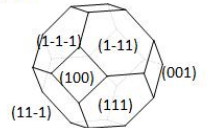

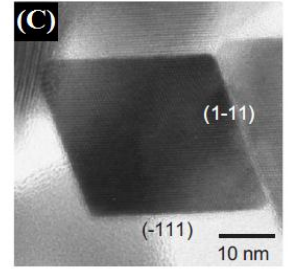

(D)

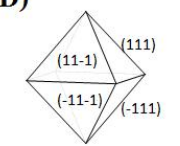

Fig. 3 HRTEM images of magnetic crystals synthesized by partial oxidation. The crystals synthesized in the presence (A) or absence (B) of Mms6. (C) and (D) are model crystal structures. magnetic particles and is involved in the formation of a $\left\{\begin{array}{lll}1 & 0 & 0\end{array}\right\}$ face. From this experiment, the chemical synthesis of magnetic particles using Mms6 is now thought to be a useful method for obtaining highly ordered magnetic particles under ordinary temperature and pressure conditions.

Moreover, the size and shape of the magnetite particles — synthesized using peptides mimicking Mms6 - could be manually controlled [14]. Four types of peptides were prepared for this examination. M6A was a putative iron-binding site of Mms6, which consisted of 12 amino acids (DIESAQSDEEVE). The peptide, whose acidic amino acids of M6A were replaced with lysine, was termed M6B. GLM6A and GLM6B were designed by attaching the GL repeated sequences to each $\mathrm{N}$-terminal of M6A and M6B, respectively. TEM analysis revealed that the magnetic particles synthesized by partial oxidation with Mms6, M6A, or GLM6A exhibited cubo-octahedral morphologies, such as the particles produced in the bacteria, while the morphologies of the magnetic particles synthesized with M6B or GLM6B were octahedron. Furthermore, the localization analysis of the M6A peptide on the synthesized magnetic particles suggested that the acidic amino acid sequence of the Mms6 C-terminal region binds to the surface of magnetic particles and plays an important role in the formation of cubo-octahedral morphologies.

The Mms6 protein was further applied to synthesize magnetic particles using cobalt, which does not naturally occur in magnetotactic bacteria [15-16]. In the presence of the Mms6 peptide and cobalt, shape-specific magnetic particles were synthesized. The cobalt ferrite particles formed in the presence of Pluronic-conjugated Mms6 peptide exhibited a very high coercivity of approximately $900 \mathrm{mT}$ at $5 \mathrm{~K}$ (compared to $500 \mathrm{mT}$ for the protein-free Pluronic) [15]. The cobalt-doping magnetic particles that were synthesized with Mms6 showed a single magnetic hysteresis with high coercivity (approximately $480 \mathrm{mT}$ at $10 \mathrm{~K}$ ) [16]. Moreover, Mms6 was applied to the synthesis of magnetic particles on 
the substrate. At the site of Mms6 attachment, the deposition of magnetic particles was observed by scanning electron microscope [17]. Similarly, the patterned magnetic-particle array was also constructed [18]. These applications suggest that Mms6 is useful in building highly ordered magnetic devices.

\section{FUNCTIONAL ANALYSIS OF THE MMS6 PROTEIN IN MAGNETOTACTIC BACTERIA}

Although the in vitro synthesis of magnetic particles by Mms6, and its subsequent applications, has been under intense investigation, the actual mechanism controlling magnetite size and morphology by Mms6 in the magnetotactic bacteria remains unknown. In order to understand the function of Mms6 in vivo, the mms6 gene deletion mutant (4mms6) was constructed by homologous recombination [19]. When the mms 6 gene was deleted from the AMB-1 wild-type genome, $\Delta m m s 6$ synthesized small particles in their cells. The average particle size $(27.4 \pm 8.9 \mathrm{~nm})$ and shape factor $(0.74 \pm 0.23 \mathrm{~nm})$ for $\Delta m m s 6$ were significantly different from the wild type, with $48.3 \pm 12.5 \mathrm{~nm}$ particle size and $0.92 \pm 0.16 \mathrm{~nm}$ shape factor. However, the number of magnetic particles in $\Delta m m s 6$ remained unaffected. These results indicate that Mms6 does not function in the nucleation step of

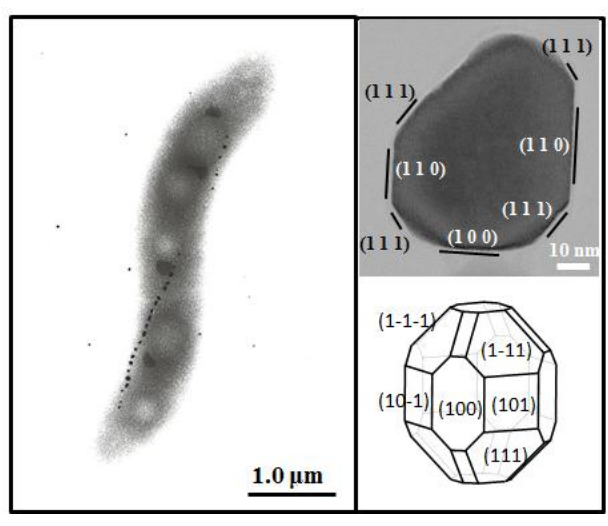

Fig. 4 HRTEM images and ideal models of magnetic crystals synthesized in $\Delta m m s 6$ magnetite biomineralization. For further morphological analysis of magnetic crystals in $4 m m s 6$, the crystals were extracted from the mutant and observed by high-resolution TEM. The crystals formed in the $\Delta m m s 6$ exhibited $\{210\},\{2$ $\left.\begin{array}{ll}1 & 1\end{array}\right\}$, and $\left\{\begin{array}{lll}3 & 1 & 1\end{array}\right\}$ faces, while the wild-type strain synthesized cubo-octahedral crystals with $\left\{\begin{array}{llllll}1 & 1 & 1\end{array}\right\}$ and $\left\{\begin{array}{lllll}1 & 0 & 0\end{array}\right\}$ faces (Fig. 4). These results indicate that Mms6 is directly involved in the size and morphological control of magnetic particles. Interestingly, the profile of the proteins surrounding the magnetic particles in $4 m m s 6$ showed the absence of Mms6 and a decrease in the expression levels of Mms5, Mms7, and Mms13. These results indicate that these proteins may interact with Mms6 and co-localize on the magnetic particles. The results of the $\Delta m m s 6$ phenotype, which included small and elongated particles, suggest that Mms6 may accelerate the growth of magnetic crystals during the process of crystal formation. Furthermore, Mms6 may interact with other Mms proteins and regulate crystal morphology.

Other studies have suggested the existence of proteins involved in the control of particle size and morphology. In the Magnetospirillum gryphiswaldense MSR-1 strain, the mamGFDC operon was deleted from its genome [20]. MamD and MamC are homologies of Mms7 and Mms13, respectively, in strain AMB-1. The $\triangle m a m G F D C$ produced slightly small magnetic particles. This result indicates that MamGFDC is involved in the control of the size of magnetic particles in strain MSR-1. Since $\Delta m m s 13$, which was constructed from strain AMB-1, also synthesized small magnetic particles, it appears that Mms13 may play a similar role (i.e., to that of MamGFDC) in strain AMB-1 [21]. The $\Delta m m s F$ were found to produce small and elongated particles, such as $\Delta m m s 6$ [22]. Although these various phenotypes of the gene-deletion mutants suggest a complicated pathway in regulating magnetic particles, the function of these genes are not known.

From the crystallographic analysis of the magnetic particles in $\Delta m m s 6$, we determined that Mms6 controls the formation of the crystal faces in the magnetotactic bacteria. The $\left\{\begin{array}{lll}1 & 1 & 0\end{array}\right\}$ crystal face observed only on the surfaces of the crystals in the $\Delta m m s 6$ had a higher surface energy than the $\left\{\begin{array}{lll}1 & 0 & 0\end{array}\right\}$ and $\left\{\begin{array}{lll}1 & 1 & 1\end{array}\right\}$ crystal faces. Therefore, the $\left\{\begin{array}{lllll}1 & 1 & 0\end{array}\right\}$ faces do not form on mature magnetic crystal in the wild type because they disappear with crystal growth. The $\left\{\begin{array}{llll}1 & 0\end{array}\right\}$ crystal face of the crystals in $\Delta m m s 6$ indicates that crystal growth had not been fully completed because of the absence of Mms6. A previous study suggested that Mms6 and mimicking peptides in an iron solution may stabilize or accelerate the iron hydroxide formation process. This idea complements the results obtained from $\Delta m m s 6$. Herein, we describe a putative role for Mms6 in the process of crystal formation in cells. In this process, iron hydroxides $\left[\mathrm{Fe}(\mathrm{OH})_{2}\right]$ first aggregate and nucleate into larger amorphous cores. The cores repeat aggregation and dissolution in a solution due to their instability and then grow into magnetites. At this time, Mms6 stabilizes the specific crystal faces by interacting with the surface of the crystals. Thus, the region of the crystal surface stabilized by Mms6 is not dissolved through the process of crystal growth. Consequently, the mature crystals have the crystal faces affected by Mms6. In this process, Mms6 controls crystal 
morphology by forming specific crystal faces.

\section{CONCLUSION}

Because Mms6 is isolated on the surface of magnetic particles in AMB-1, it is considered to directly associate with magnetites by the acidic amino acid of the C-terminal region. This characteristic of Mms6 was applied to the chemical synthesis of magnetic particles under normal temperature and pressure conditions. We succeeded in controlling the size and morphology of magnetic particles by using Mms6 and mimicking peptides. From the HRTRM analysis, the $\left\{\begin{array}{lll}1 & 0 & 0\end{array}\right\}$ crystal faces were observed on the magnetic particles synthesized with Mms6. This result indicates that Mms6 specifically interacts with the $\left\{\begin{array}{lll}1 & 0 & 0\end{array}\right\}$ face. The spherical morphology of the particles synthesized with Mms6 was identical to that of the particles synthesized in the presence of the Mms6 C-terminus peptide. The acidic amino acids, including those in the Mms6 C-terminus, were suggested to be involved in the formation of the $\left\{\begin{array}{lll}1 & 0 & 0\end{array}\right\}$ crystal face. To understand the role of Mms6 in magnetotactic bacteria, an mms6 gene-deletion mutant was constructed. This mutant produced small and elongated crystals, which have unobserved crystal faces in the wild-type strain. To our knowledge, this study is the first report on a protein that directly acts as a morphological regulator of magnetic particles in vivo. From in vivo and in vitro analyses, the function of Mms6 appears to involve the stabilization of the $\left\{\begin{array}{lll}1 & 0 & 0\end{array}\right\}$ crystal face. During the process of synthesizing magnetic particles, the acidic amino acids of the Mms6 C-terminal region interact with the iron ion of the $\{1$ $\left.\begin{array}{lll}0 & 0\end{array}\right\}$ face. The stabilized $\left\{\begin{array}{lll}1 & 0 & 0\end{array}\right\}$ face is finally formed on the surface of the magnetic crystal. Since Mms6 is able to control magnetic crystal morphology in vivo, further applications of Mms6 are expected to synthesize magnetic materials by magnetotactic bacteria.

\section{ACKNOWLEDGMENTS}

This work was funded in part by a Grant-in-Aid for Specially Promoted Research, No. 23226016 from the Scientific Research for the Ministry of Education, Culture, Sports, Science and Technology of Japan, This work was also funded in part by a Grant-in-Aid for Scientific Research on Innovative Areas (no. 2206) from the Ministry of Education, Culture, Sports, Science and Technology, Japan.

\section{REFERENCES}

[1] R. B. Frankel, R. P. Blakemore and R. S. Wolfe, Science. 203, 1355 (1979).

[2] R. P. Blakemore, R. B. Frankel and A. J. Kalmijn, Nature. 286, 384 (1980).

[3] T. Matsunaga, T. Sakaguchi and F. Tadokoro, Appl. Microbiol. Biotechnol. 35, 651 (1991).

[4] K. H. Schleifer, D. Schuler, S. Spring, M. Weizenegger, R. Amann, W. Ludwig, et al., Syst. Appl. Microbiol. 14, 379 (1991).

[5] F. C. Meldrum, S. Mann, B. R. Heywood, R. B. Frankel, and D. A. Bazylinski, Proc. R. Soc. Lond. B. Biol. Sci. 251, 237 (1993).

[6] T. Sakaguchi, J. G. Burgess and T. Matsunaga, Nature. 365, 47 (1993).

[7] S. Spring, R. Amann, W. Ludwig, K. H. Schleifer, H. van. Gemerden and N. Petersen, Appl. Environ. Microbiol. 59, 2397 (1993).

[8] A. Arakaki, J. Webb, and T. Matsunaga, J. Biol. Chem. 278, 8745 (2003).

[9] H. Nishio, T. Takahashi, H. Taguchi, S. Kamiya and T. Matsunaga, J. Phys. IV France. 7, C1-663 (1997).

[10] T. Matsunaga, Y. Okamura, Y. Fukuda, A. T. Wahyudi, Y. Murase and H. Takeyama, DNA. Res. 12, 157 (2005).

[11] M. Tanaka, Y. Okamura, A. Arakaki, T. Tanaka, H. Takeyama and T. Matsunaga, Proteomics. 6, 5234 (2006).

[12] T. Suzuki, Y. Okamura, R. J. Calugay, H. Takeyama and T. Matsunaga, J. Bacteriol. 188, 2275 (2006).

[13] Y. Amemiya, A. Arakaki, S. S. Staniland, T. Tanaka and T. Matsunaga, Biomaterials. 28, 5381 (2007).

[14] A. Arakaki, F. Masuda, Y. Amemiya, T. Tanaka and T. Matsunaga, J. Colloid. Interface. Sci. 343, 65 (2010).

[15] T. Prozorov, P. Palo, L. Wang, M. Nilsen-Hamilton, D. Jones, D. Orr, et al., ACS. Nano. 1, 228 (2007).

[16] J. M. Galloway, A. Arakaki, F. Masuda, T. Tanaka, T. Matsunaga and S. S. Staniland, J. Mater. Chem. 21, 15244 (2011).

[17] A. Arakaki, F. Masuda, and T. Matsunaga, "Mater. Res. Soc. Symp. Proc., San Francisco, United States of America", Materials Research Society 2009, p. 1187.

[18] J. M. Galloway, J. P. Bramble, A. E. Rawlings, G. Burnell, S. D. Evans and S. S. Staniland, Small. 8, 204 (2012).

[19] M. Tanaka, E. Mazuyama, A. Arakaki and T. Matsunaga, J. Biol. Chem. 286, 6386 (2010). 
[20] A. Scheffel, A. Gärdes, K. Grünberg, G. Wanner and D. Schüler, J. Bacteriol. 190, 377 (2008).

[21] Y. Kanetsuki, M. Tanaka, T. Tanaka, T. Matsunaga and T. Yoshino, Biochem. Biophys. Res. Commun. 426, 7 (2012).

[22] D. Murat, V. Falahati, L. Bertinetti, R. Csencsits, A. Körnig, K. Downing, et al., Mol. Microbiol. 85, 684 (2012). 